\title{
Bilinear Localization Operators on Modulation Spaces
}

\author{
Nenad Teofanov (iD \\ Department of Mathematics and Informatics, University of Novi Sad, Novi Sad, Serbia \\ Correspondence should be addressed to Nenad Teofanov; nenad.teofanov@dmi.uns.ac.rs
}

Received 30 October 2017; Accepted 17 December 2017; Published 15 January 2018

Academic Editor: Kasso A. Okoudjou

Copyright (C) 2018 Nenad Teofanov. This is an open access article distributed under the Creative Commons Attribution License, which permits unrestricted use, distribution, and reproduction in any medium, provided the original work is properly cited.

We introduce a class of bilinear localization operators and show how to interpret them as bilinear Weyl pseudodifferential operators. Such interpretation is well known in linear case whereas in bilinear case it has not been considered so far. Then we study continuity properties of both bilinear Weyl pseudodifferential operators and bilinear localization operators which are formulated in terms of a modified version of modulation spaces.

\section{Introduction}

Localization operators were introduced by Berezin in the study of general Hamiltonians related to quantization problem in quantum mechanics [1]. In signal analysis they are related to the localization technique proposed by SlepianPolak-Landau; see, for example, a survey article [2]. Thereafter, a more detailed study of localization in phase space together with basic facts on localization operators and their applications in optics and signal analysis was given by Daubechies in [3]. That paper initiated further study of the topic. Daubechies studied localization operators $A_{a}^{\varphi}=A_{a}^{\varphi_{1}, \varphi_{2}}$ on $L^{2}\left(\mathbb{R}^{d}\right)$ (see Section 2 for the definition) with Gaussian windows

$$
\varphi_{1}(t)=\varphi_{2}(t)=\pi^{-1 / 4} \exp \left(-\frac{t^{2}}{2}\right),
$$

$$
\text { radial symbol } a \in L^{1}\left(\mathbb{R}^{d}\right) \text {. }
$$

Such operators are named Daubechies' operators afterwards. The eigenfunctions of Daubechies' operators are Hermite functions:

$$
\begin{array}{r}
h_{n}(t) \\
=(-1)^{n} \pi^{-1 / 4}\left(2^{n} n !\right)^{-1 / 2} \exp \left(\frac{t^{2}}{2}\right)\left(\exp \left(-t^{2}\right)\right)^{(n)}, \\
t \in \mathbb{R}^{d}, n=0,1, \ldots,
\end{array}
$$

and the eigenvalues can be explicitly calculated. This is an important issue in applications (cf. [4]).

For related inverse problem in the case of simply connected localization domain $\Omega$, we refer to [5] where it is proved that if one of the eigenfunctions of Daubechies' operator is a Hermite function, then $\Omega$ is a disc centered at the origin.

An exposition of different quantization theories and connection between localization operators and Toeplitz operators is given in, for example, $[6,7]$. The problem of quantization served as a motivation for the study of localization operators on $L^{p}(G), 1 \leq p \leq \infty$, where $G$ is a locally compact group; see [8]. There one can also find a product formula and Schatten-von Neumann properties of localization operators; see also [9].

Since the beginning of the XXI century, localization operators in the context of modulation spaces were studied by many authors (cf. [10-23]). See also the references given there.

Our results are related to, but different from, investigations given in [15] due to the difference in definitions of bilinear localization operators; see Remark 2. Moreover, instead of standard modulation spaces $M^{p, q}$ observed in [15], continuity properties are here formulated in terms of a modified version of modulation spaces denoted by $\mathscr{M}_{s, t}^{p, q}$; see Definition 5. Also, in contrast to [15] in this paper we do not observe multilinear version of localization operators, since we use sharp convolution estimates for modulation spaces given in [24]; see Theorems 8 and 9. These results are well 
suited for the study of bilinear operators, but their extension to multilinear case is a challenging problem.

One of the key ingredients in our investigations is the interpretation of bilinear localization operators as bilinear Weyl pseudodifferential operators, $\Psi D O$ for short. Indeed, results on multilinear Kohn-Nirenberg $\Psi$ DOs from [10-12] served as a background for the continuity properties of multilinear Kohn-Nirenberg localization operators given in [15]. Here we consider the so-called Weyl correspondence instead and obtain continuity properties (Theorems 15 and 16) analogous to [15, Theorems 4.5 and 4.8] when restricted to bilinear operators.

The paper can be summarized as follows. We first introduce bilinear localization operators, bilinear Weyl $\Psi$ DOs, and bilinear Wigner transform and show how they are connected; see Section 2. In particular, we prove that bilinear localization operator can be interpreted as bilinear $\Psi D O$, Theorem 4. In Section 3 we recall the definition and basic properties of modulation spaces and introduce their convenient modified version $\mathscr{M}_{s, t}^{p, q}$ (see Definition 5 for details). Then we recall convolution estimates from [24] which will be used in the proof of main results of the paper given in Section 4. These results are boundedness of bilinear $\Psi$ DOs on $\mathscr{M}_{s, t}^{p, q}$ (Theorem 14) and sufficient and necessary conditions for boundedness of bilinear localization operators on $\mathscr{M}_{s, t}^{p, q}$ (Theorems 15 and 16, resp.).

Notation. The Schwartz class is denoted by $\mathcal{S}\left(\mathbb{R}^{d}\right)$ and the space of tempered distributions by $\mathcal{S}^{\prime}\left(\mathbb{R}^{d}\right)$. We use the brackets $\langle f, g\rangle$ to denote the extension of the inner product $\langle f, g\rangle=\int f(t) \overline{g(t)} d t$ on $L^{2}\left(\mathbb{R}^{d}\right)$ to any pair of dual spaces. The Fourier transform is normalized to be $\widehat{f}(\omega)=\mathscr{F} f(\omega)=$ $\int f(t) e^{-2 \pi i t \omega} d t$. The involution $f^{*}$ is $f^{*}(\cdot)=\overline{f(-\cdot)}$, and the convolution of $f$ and $g$ is given by $f * g(x)=\int f(x-$ $y) g(y) d y$, when the integral exists.

We denote by $\langle\cdot\rangle^{s}$ the polynomial weights

$$
\langle(x, \omega)\rangle^{s}=\left(1+|x|^{2}+|\omega|^{2}\right)^{s / 2}, \quad(x, \omega) \in \mathbb{R}^{2 d}, s \in \mathbb{R}
$$

and $\langle x\rangle=\left\langle 1+|x|^{2}\right\rangle^{1 / 2}$, when $x \in \mathbb{R}^{d}$.

We use the notation $A \leqslant B$ to indicate that $A \leq c B$ for a suitable constant $c>0$, whereas $A \asymp B$ means that $c^{-1} A \leq$ $B \leq c A$ for some $c \geq 1$.

\section{Bilinear Localization Operators}

To define localization operators we start with the short-time Fourier transform, a time-frequency representation related to Feichtinger's modulation spaces $[25,26]$.

Let $\mathcal{S}^{(1)}\left(\mathbb{R}^{d}\right)$ be the Gelfand-Shilov type space of analytic functions given by

$$
\begin{aligned}
f & \in \mathcal{S}^{(1)}\left(\mathbb{R}^{d}\right) \Longleftrightarrow \\
\left\|f(x) e^{h \cdot|x|}\right\|_{L^{\infty}} & <\infty,
\end{aligned}
$$

$$
\left\|\widehat{f}(\omega) e^{h \cdot|\omega|}\right\|_{L^{\infty}}<\infty
$$

$\forall h>0$.

If $f \in \mathcal{S}^{(1)}\left(\mathbb{R}^{d}\right)$, then $f$ extends to a holomorphic function $f(x+i y)$ in the strip $\left\{x+i y \in \mathbb{C}^{d}:|y|<T\right\}$ for a suitable $T>0[27,28]$. The dual space of $\mathcal{S}^{(1)}\left(\mathbb{R}^{d}\right)$ will be denoted by $\left(\mathcal{S}^{(1)}\right)^{\prime}\left(\mathbb{R}^{d}\right)$.

Translation and modulation operators are given by

$$
\begin{aligned}
T_{x} f(\cdot) & =f(\cdot-x), \\
M_{\omega} f(\cdot) & =e^{2 \pi i \omega \cdot} f(\cdot)
\end{aligned}
$$

$$
x, \omega \in \mathbb{R}^{d} .
$$

The short-time Fourier transform (STFT in the sequel) of $f \in \mathcal{S}^{(1)}\left(\mathbb{R}^{d}\right)$ with respect to the window $g \in \mathcal{S}^{(1)}\left(\mathbb{R}^{d}\right) \backslash 0$ is given by

$$
\begin{aligned}
V_{g} f(x, \omega) & =\left\langle f, M_{\omega} T_{x} g\right\rangle \\
& =\int_{\mathbb{R}^{d}} f(t) \overline{g(t-x)} e^{-2 \pi i \omega t} d t .
\end{aligned}
$$

The map $(f, g) \mapsto V_{g} f$ from $\mathcal{S}^{(1)}\left(\mathbb{R}^{d}\right) \otimes \mathcal{S}^{(1)}\left(\mathbb{R}^{d}\right)$ to $\delta^{(1)}\left(\mathbb{R}^{2 d}\right)$ extends uniquely to a continuous operator from $\left(\mathcal{S}^{(1)}\right)^{\prime}\left(\mathbb{R}^{d}\right) \otimes\left(\mathcal{S}^{(1)}\right)^{\prime}\left(\mathbb{R}^{d}\right)$ to $\left(\mathcal{S}^{(1)}\right)^{\prime}\left(\mathbb{R}^{2 d}\right)$ by duality.

Moreover, for a fixed $g \in \mathcal{S}^{(1)}\left(\mathbb{R}^{d}\right) \backslash 0$ the following characterization holds:

$$
\begin{gathered}
f \in \mathcal{S}^{(1)}\left(\mathbb{R}^{d}\right) \Longleftrightarrow \\
V_{g} f \in \mathcal{S}^{(1)}\left(\mathbb{R}^{2 d}\right) .
\end{gathered}
$$

We refer to [20, 21, 29-31] for the proof and more details on STFT in other spaces of Gelfand-Shilov type.

The localization operator $A_{a}^{\varphi_{1}, \varphi_{2}}$ with symbol $a \in L^{2}\left(\mathbb{R}^{2 d}\right)$ and windows $\varphi_{1}, \varphi_{2} \in L^{2}\left(\mathbb{R}^{d}\right)$ is given by

$$
\begin{aligned}
& A_{a}^{\varphi_{1}, \varphi_{2}} f(t) \\
& \quad=\int_{\mathbb{R}^{2 d}} a(x, \omega) V_{\varphi_{1}} f(x, \omega) M_{\omega} T_{x} \varphi_{2}(t) d x d \omega, \\
& \quad f \in L^{2}\left(\mathbb{R}^{d}\right) .
\end{aligned}
$$

Next, we define bilinear localization operators as follows.

Definition 1. Let $f_{1}, f_{2} \in \mathcal{S}^{(1)}\left(\mathbb{R}^{d}\right)$ and $\vec{f}=\left(f_{1}, f_{2}\right)$. The bilinear localization operator $A_{a}^{\varphi}$ with symbol $a \in \mathcal{S}^{(1)^{\prime}}\left(\mathbb{R}^{4 d}\right)$ and window $\varphi=(\vec{\varphi}, \vec{\phi})=\left(\varphi_{1}, \varphi_{2}, \phi_{1}, \phi_{2}\right)$, where $\varphi_{j}, \phi_{j} \in$ $\delta^{(1)}\left(\mathbb{R}^{d}\right), j=1,2$, is given by

$$
\begin{aligned}
& A_{a}^{\varphi} \vec{f}(t)=\int_{\mathbb{R}^{2 d}} a(x, \omega) V_{\varphi_{1}} f_{1}\left(x_{1}, \omega_{1}\right) V_{\varphi_{2}} f_{2}\left(x_{2}, \omega_{2}\right) \\
& \cdot M_{\omega_{1}} T_{x_{1}} \phi_{1}\left(t_{1}\right) M_{\omega_{2}} T_{x_{2}} \phi_{2}\left(t_{2}\right) d x d \omega,
\end{aligned}
$$

where $x=\left(x_{1}, x_{2}\right), \omega=\left(\omega_{1}, \omega_{2}\right), t=\left(t_{1}, t_{2}\right)$, and $x_{1}, x_{2}$, $\omega_{1}, \omega_{2}, t_{1}$, and $t_{2} \in \mathbb{R}^{d}$. 
Remark 2. Let $\mathscr{R}$ denote the trace mapping that assigns to each function $F$ defined on $\mathbb{R}^{2 d}$ a function defined on $\mathbb{R}^{d}$ by the formula

$$
\mathscr{R}:\left.F \longmapsto F\right|_{t_{1}=t_{2}} \quad t_{1}, t_{2} \in \mathbb{R}^{d} .
$$

Then, $\mathscr{R} A_{a}^{\varphi}$ is the bilinear operator given in $[15$, Definition $1.1]$.

In order to give an interpretation of bilinear operators in the weak sense, we introduce the following notation. Let there be given $\varphi_{1}, \varphi_{2}, f_{1}$, and $f_{2} \in \mathcal{S}^{(1)}\left(\mathbb{R}^{d}\right)$. Then

$$
\begin{aligned}
& V_{\varphi_{1} \otimes \varphi_{2}}\left(f_{1} \otimes f_{2}\right)(x, \omega)=\int_{\mathbb{R}^{2 d}} f_{1}\left(t_{1}\right) f_{2}\left(t_{2}\right) \\
& \cdot \overline{M_{\omega_{1}} T_{x_{1}} \varphi_{1}\left(t_{1}\right) M_{\omega_{2}} T_{x_{2}} \varphi_{2}\left(t_{2}\right)} d t_{1} d t_{2} \\
& \quad=\int_{\mathbb{R}^{2 d}}\left(f_{1} \otimes f_{2}\right)(t) \overline{\left(M_{\omega_{1}} T_{x_{1}} \varphi_{1} \otimes M_{\omega_{2}} T_{x_{2}} \varphi_{2}\right)(t)} d t,
\end{aligned}
$$

where $x=\left(x_{1}, x_{2}\right), \omega=\left(\omega_{1}, \omega_{2}\right), t=\left(t_{1}, t_{2}\right)$, and $x_{1}, x_{2}, \omega_{1}, \omega_{2}, t_{1}$, and $t_{2} \in \mathbb{R}^{d}$. Thus, the weak definition of (9) is given by

$$
\begin{aligned}
\left\langle A_{a}^{\varphi} \vec{f}, \vec{g}\right\rangle & =\left\langle a V_{\varphi_{1} \otimes \varphi_{2}}\left(f_{1} \otimes f_{2}\right), V_{\phi_{1} \otimes \phi_{2}}\left(g_{1} \otimes g_{2}\right)\right\rangle \\
& =\left\langle a, \overline{V_{\varphi_{1} \otimes \varphi_{2}}\left(f_{1} \otimes f_{2}\right)} V_{\phi_{1} \otimes \phi_{2}}\left(g_{1} \otimes g_{2}\right)\right\rangle,
\end{aligned}
$$

and $f_{1}, f_{2}, g_{1}$, and $g_{2} \in \mathcal{S}^{(1)}\left(\mathbb{R}^{d}\right)$. The brackets can be interpreted as suitable duality between a pair of dual spaces. Thus, $A_{a}^{\varphi}$ is a well-defined continuous operator from $\mathcal{S}^{(1)}\left(\mathbb{R}^{d}\right) \otimes$ $\mathcal{S}^{(1)}\left(\mathbb{R}^{d}\right)$ to $\left(\mathcal{S}^{(1)}\right)^{\prime}\left(\mathbb{R}^{4 d}\right)$.

Next, we introduce a class of bilinear Weyl pseudodifferential operators and use the Wigner transform to provide appropriate interpretation of bilinear localization operators as bilinear Weyl pseudodifferential operators.

Let $\sigma \in \mathcal{S}^{(1)}\left(\mathbb{R}^{2 d}\right)$. Then the Weyl pseudodifferential operator $L_{\sigma}$ with the Weyl symbol $\sigma$ can be defined as the oscillatory integral:

$$
\begin{array}{r}
L_{\sigma} f(x)=\iint \sigma\left(\frac{x+y}{2}, \omega\right) f(y) e^{2 \pi i(x-y) \cdot \omega} d y d \omega, \\
f \in \mathcal{S}^{(1)}\left(\mathbb{R}^{d}\right) .
\end{array}
$$

This definition extends to each $\sigma \in \mathcal{S}^{(1)^{\prime}}\left(\mathbb{R}^{2 d}\right)$, so that $L_{\sigma}$ is a continuous mapping from $\mathcal{S}^{(1)}\left(\mathbb{R}^{2 d}\right)$ to $\mathcal{S}^{(1)^{\prime}}\left(\mathbb{R}^{2 d}\right)$. If

$$
\begin{array}{r}
W(f, g)(x, \omega)=\int f\left(x+\frac{t}{2}\right) \overline{g\left(x-\frac{t}{2}\right)} e^{-2 \pi i \omega t} d t, \\
f, g \in \mathcal{S}^{(1)}\left(\mathbb{R}^{d}\right),
\end{array}
$$

denotes the Wigner transform, also known as the crossWigner distribution, then the following formula holds:

$$
\left\langle L_{\sigma} f, g\right\rangle=\langle\sigma, W(g, f)\rangle, \quad f, g \in \mathcal{S}^{(1)}\left(\mathbb{R}^{d}\right),
$$

for each $\sigma \in \mathcal{S}^{(1)^{\prime}}\left(\mathbb{R}^{2 d}\right)$; see, for example, $[26,32,33]$.
By analogy with (13) we define the bilinear Weyl pseudodifferential operator as follows:

$$
\begin{gathered}
L_{\sigma}(\vec{f})(x)=\int_{\mathbb{R}^{4 d}} \sigma\left(\frac{x+y}{2}, \omega\right) f_{1}\left(y_{1}\right) f_{2}\left(y_{2}\right) \\
\cdot e^{2 \pi i \mathscr{F}(x-y) \cdot \omega} d y d \omega, \quad x \in \mathbb{R}^{2 d},
\end{gathered}
$$

where $\sigma \in \mathcal{S}^{(1)^{\prime}}\left(\mathbb{R}^{4 d}\right), f_{1}, f_{2} \in \mathcal{S}^{(1)}\left(\mathbb{R}^{d}\right)$, and $\vec{f}=\left(f_{1}, f_{2}\right)$. Here $\mathscr{I}$ denotes the identity matrix in $2 d$ (e.g., if $d=2$, then $\left.\mathscr{I}(x-y) \cdot \omega=\omega_{1}\left(x_{1}-y_{1}\right)+\omega_{2}\left(x_{2}-y_{2}\right)\right)$.

To give the interpretation of (15) in the context of bilinear YDOs we introduce the bilinear version of (14) as follows. Let $f_{1}, f_{2}, g_{1}$, and $g_{2} \in \mathcal{S}^{(1)}\left(\mathbb{R}^{d}\right), \vec{f}=\left(f_{1}, f_{2}\right)$, and $\vec{g}=\left(g_{1}, g_{2}\right)$. Then the bilinear Wigner transform $W(\vec{f}, \vec{g})$ is given by

$$
\begin{aligned}
& W(\vec{f}, \vec{g})(x, \omega)=W\left(f_{1}, g_{1}\right)\left(x_{1}, \omega_{1}\right) \otimes W\left(f_{2}, g_{2}\right) \\
& \cdot\left(x_{2}, \omega_{2}\right)=\int_{\mathbb{R}^{2 d}} f_{1}\left(x_{1}+\frac{t_{1}}{2}\right) \\
& \quad \overline{g_{1}\left(x_{1}-\frac{t_{1}}{2}\right)} f_{2}\left(x_{2}+\frac{t_{2}}{2}\right) \\
& \quad \frac{g_{2}\left(x_{2}-\frac{t_{2}}{2}\right)}{2} e^{-2 \pi i \mathscr{F} \omega t} d t,
\end{aligned}
$$

and $t=\left(t_{1}, t_{2}\right) \in \mathbb{R}^{d} \times \mathbb{R}^{d}$. It is easy to see that $W(\vec{f}, \vec{g}) \in$ $\mathcal{S}^{(1)}\left(\mathbb{R}^{4 d}\right)$.

Lemma 3. Let $\sigma \in \mathcal{S}^{(1)}\left(\mathbb{R}^{4 d}\right)$ and $f_{1}, f_{2}, g_{1}$, and $g_{2} \in$ $\delta^{(1)}\left(\mathbb{R}^{d}\right)$. Then $L_{\sigma}$ given by (16) extends to a continuous map from $\mathcal{S}^{(1)}\left(\mathbb{R}^{d}\right) \otimes \mathcal{S}^{(1)}\left(\mathbb{R}^{d}\right)$ to $\mathcal{S}^{(1)^{\prime}}\left(\mathbb{R}^{4 d}\right)$ and the following formula holds:

$$
\left\langle L_{\sigma} \vec{f}, \vec{g}\right\rangle=\langle\sigma, W(\vec{g}, \vec{f})\rangle
$$

Proof. The proof follows by the straightforward calculation:

$$
\begin{aligned}
& \langle\sigma, W(\vec{g}, \vec{f})\rangle=\int_{\mathbb{R}^{4 d}} \sigma(x, \omega) W(\vec{f}, \vec{g}) \\
& \cdot(x, \omega) d x d \omega=\int_{\mathbb{R}^{6 d}} \sigma(x, \omega) f_{1}\left(x_{1}+\frac{t_{1}}{2}\right) \\
& \cdot \overline{g_{1}\left(x_{1}-\frac{t_{1}}{2}\right)} f_{2}\left(x_{2}+\frac{t_{2}}{2}\right) \\
& \cdot \frac{g_{2}\left(x_{2}-\frac{t_{2}}{2}\right)}{2} e^{-2 \pi i \mathscr{F} \omega t} d t d x d \omega \\
& =\int_{\mathbb{R}^{6 d}} \sigma\left(\frac{u+v}{2}, \omega\right) f_{1}\left(v_{1}\right) \overline{g_{1}\left(u_{1}\right)} f_{2}\left(v_{2}\right) \\
& \cdot \frac{g_{2}\left(u_{2}\right)}{-2 \pi i \mathscr{F}(u-v) \omega} d u d v d \omega=\left\langle\sigma\left(\frac{u+v}{2}, \omega\right)\right. \\
& \left.\cdot\left(f_{1} \otimes f_{2}\right)(v) e^{2 \pi i \mathscr{F}(u-v) \omega},\left(g_{1} \otimes g_{2}\right)(u)\right\rangle \\
& =\left\langle L_{\sigma} \vec{f}, \vec{g}\right\rangle,
\end{aligned}
$$


where we used $W(\vec{g}, \vec{f})=\overline{W(\vec{f}, \vec{g})}$ and the change of variables $u=x+t / 2$ and $v=x-t / 2$. This extends to each $\sigma \in \mathcal{S}^{(1)^{\prime}}\left(\mathbb{R}^{4 d}\right)$, since $W(\vec{f}, \vec{g}) \in \mathcal{S}^{(1)}\left(\mathbb{R}^{4 d}\right)$ when $f_{1}, f_{2}, g_{1}$, and $g_{2} \in \mathcal{S}^{(1)}\left(\mathbb{R}^{d}\right)$.

The so-called Weyl connection between the set of linear localization operators and Weyl $\Psi$ DOs is well known; we refer to, for example, [21, 32, 34]. The proof of the following Weyl connection between the set of bilinear localization operators and corresponding bilinear Weyl $\Psi$ DOs is based on the kernel theorem for Gelfand-Shilov spaces (see, e.g., [20,35]) and direct calculations. Since the proof is quite technical we present it in the separate Section 5. The conclusion of Theorem 4 is that, as in the linear case, the bilinear localization operators can be viewed as a subclass of the bilinear Weyl $\Psi$ DOs.

Theorem 4. Let there be given $a \in \mathcal{S}^{(1)^{\prime}}\left(\mathbb{R}^{2 d}\right)$ and let $\vec{\varphi}=$ $\left(\varphi_{1}, \varphi_{2}\right)$ and $\vec{\phi}=\left(\phi_{1}, \phi_{2}\right)$, where $\varphi_{j} \in \mathcal{S}^{(1)}\left(\mathbb{R}^{d}\right), j=1,2$. Then the localization operator $A_{a}^{\varphi}$ is the Weyl pseudodifferential operator with the Weyl symbol

$$
\sigma=a * W(\vec{\phi}, \vec{\varphi})=a *\left(W\left(\phi_{1}, \varphi_{1}\right) \otimes W\left(\phi_{2}, \varphi_{2}\right)\right) .
$$

Therefore, if $\vec{f}=\left(f_{1}, f_{2}\right), f_{1}, f_{2}, \in \mathcal{S}^{(1)^{\prime}}\left(\mathbb{R}^{d}\right)$, and $\vec{g}=$ $\left(g_{1}, g_{2}\right), g_{1}, g_{2}, \in \mathcal{S}^{(1)}\left(\mathbb{R}^{d}\right)$, we have

$$
\begin{aligned}
\left\langle A_{a}^{\varphi} \vec{f}, \vec{g}\right\rangle & =\left\langle L_{a * W(\vec{\phi}, \vec{\varphi})} \vec{f}, \vec{g}\right\rangle \\
& =\left\langle L_{a *\left(W\left(\phi_{1}, \varphi_{1}\right) \otimes W\left(\phi_{2}, \varphi_{2}\right)\right)} \vec{f}, \vec{g}\right\rangle .
\end{aligned}
$$

\section{Modulation Spaces}

Since we essentially use the convolution estimates for polynomially weighted modulation spaces (Theorems 8 and 9), by Theorem 7(3) below it is enough to use the duality between $\delta$ and $\mathcal{S}^{\prime}$ instead of the more general duality between $\mathcal{S}^{(1)}$ and $\mathcal{S}^{(1)^{\prime}}$. We refer to $[16,17]$ for investigations in the framework of subexponential and superexponential weights and leave the study of bilinear localization operators in that case for a separate contribution.

Modulation spaces $[25,26]$ are defined through decay and integrability conditions on STFT, which makes them suitable for time-frequency analysis and for the study of localization operators in particular. Their definition is given in terms of weighted mixed-norm Lebesgue spaces.

In general, a weight $w(\cdot)$ on $\mathbb{R}^{d}$ is a nonnegative and continuous function. By $L_{w}^{p}\left(\mathbb{R}^{d}\right)$ and $p \in[1, \infty]$, we denote the weighted Lebesgue space defined by the norm

$$
\|f\|_{L_{w}^{p}}=\|f w\|_{L^{p}}=\left(\int|f(x)|^{p} w(x)^{p} d x\right)^{1 / p},
$$

with the usual modification when $p=\infty$. When $w(x)=\langle x\rangle^{t}$, $t \in \mathbb{R}$, we use the notation $L_{t}^{p}\left(\mathbb{R}^{d}\right)$ instead.
Similarly, the weighted mixed-norm space $L_{w}^{p, q}\left(\mathbb{R}^{2 d}\right)$, $p, q \in[1, \infty]$, consists of (Lebesgue) measurable functions on $\mathbb{R}^{2 d}$ such that

$$
\begin{aligned}
& \|F\|_{L_{w}^{p, q}} \\
& \quad=\left(\int_{\mathbb{R}^{d}}\left(\int_{\mathbb{R}^{d}}|F(x, \omega)|^{p} w(x, \omega)^{p} d x\right)^{q / p} d \omega\right)^{1 / q} \\
& \quad<\infty,
\end{aligned}
$$

where $w(x, \omega)$ is a weight on $\mathbb{R}^{2 d}$.

In particular, when $w(x, \omega)=\langle x\rangle^{t}\langle\omega\rangle^{s}, s, t \in \mathbb{R}$, we use the notation $L_{w}^{p, q}\left(\mathbb{R}^{2 d}\right)=L_{t, s}^{p, q}\left(\mathbb{R}^{2 d}\right)$.

Now, modulation space $M_{s, t}^{p, q}\left(\mathbb{R}^{d}\right)$ consists of distributions whose STFT belong to $L_{t, s}^{p, q}\left(\mathbb{R}^{2 d}\right)$.

Definition 5. Let $\phi \in \mathcal{S}\left(\mathbb{R}^{d}\right) \backslash 0, s, t \in \mathbb{R}$, and $p, q \in[1, \infty]$. The modulation space $M_{s, t}^{p, q}\left(\mathbb{R}^{d}\right)$ consists of all $f \in \mathcal{S}^{\prime}\left(\mathbb{R}^{d}\right)$ such that

$$
\begin{aligned}
& \|f\|_{M_{s, t}^{p, q}} \\
& \equiv\left(\int_{\mathbb{R}^{d}}\left(\int_{\mathbb{R}^{d}}\left|V_{\phi} f(x, \omega)\langle x\rangle^{t}\langle\omega\rangle^{s}\right|^{p} d x\right)^{q / p} d \omega\right)^{1 / q} \\
& <\infty
\end{aligned}
$$

(with obvious interpretation of the integrals when $p=\infty$ or $q=\infty)$.

For the consistency, and according to (11), we denote by $\mathscr{M}_{s, t}^{p, q}\left(\mathbb{R}^{2 d}\right)$ the set of $\vec{f}=\left(f_{1}, f_{2}\right), f_{1}, f_{2} \in \mathcal{S}^{(1)^{\prime}}\left(\mathbb{R}^{d}\right)$, such that

$$
\begin{aligned}
& \|\vec{f}\|_{\mathscr{M}_{s, t}^{p, q}} \\
& \equiv\left(\int_{\mathbb{R}^{2 d}}\left(\int_{\mathbb{R}^{2 d}}\left|V_{\varphi_{1} \otimes \varphi_{2}}\left(f_{1} \otimes f_{2}\right)(x, \omega)\langle x\rangle^{t}\langle\omega\rangle^{s}\right|^{p} d x\right)^{q / p} d \omega\right)^{1 / q} \\
& <\infty .
\end{aligned}
$$

In special cases we use the usual abbreviations: $M_{0,0}^{p, p}=$ $M^{p}, M_{t, t}^{p, p}=M_{t}^{p}$, and so on.

Remark 6. Notice that the original definition given in [25] contains more general submultiplicative weights. We restrict ourselves to $w(x, \omega)=\langle x\rangle^{t}\langle\omega\rangle^{s}, s, t \in \mathbb{R}$, since the convolution and multiplication estimates which will be used later on are formulated in terms of weighted spaces with such polynomial weights. As already mentioned, weights of exponential type growth are used in the study of GelfandShilov spaces and their duals in $[16,29-31]$. We refer to [36] for a survey on the most important types of weights commonly used in time-frequency analysis.

The following theorem lists some of the basic properties of modulation spaces. We refer to $[25,26]$ for its proof. 
Theorem 7. Let $p, q, p_{j}$, and $q_{j} \in[1, \infty]$ and $s, t, s_{j}$, and $t_{j} \in$ $\mathbb{R}, j=1,2$. Then

(1) $M_{s, t}^{p, q}\left(\mathbb{R}^{d}\right)$ are Banach spaces, independent of the choice of $\phi \in \mathcal{S}\left(\mathbb{R}^{d}\right) \backslash 0$;

(2) if $p_{1} \leq p_{2}, q_{1} \leq q_{2}, s_{2} \leq s_{1}$, and $t_{2} \leq t_{1}$, then

$$
\mathcal{S}\left(\mathbb{R}^{d}\right) \subseteq M_{s_{1}, t_{1}}^{p_{1}, q_{1}}\left(\mathbb{R}^{d}\right) \subseteq M_{s_{2}, t_{2}}^{p_{2}, q_{2}}\left(\mathbb{R}^{d}\right) \subseteq \mathcal{S}^{\prime}\left(\mathbb{R}^{d}\right) ;
$$

(3) $\bigcap_{s, t} M_{s, t}^{p, q}\left(\mathbb{R}^{d}\right)=\mathcal{S}\left(\mathbb{R}^{d}\right)$ and $\bigcup_{s, t} M_{s, t}^{p, q}\left(\mathbb{R}^{d}\right)=\mathcal{S}^{\prime}\left(\mathbb{R}^{d}\right)$.

Modulation spaces include the following well-known function spaces:

(a) $M^{2}\left(\mathbb{R}^{d}\right)=L^{2}\left(\mathbb{R}^{d}\right)$.

(b) $M_{t, 0}^{2}\left(\mathbb{R}^{d}\right)=L_{t}^{2}\left(\mathbb{R}^{d}\right)$.

(c) Sobolev spaces: $M_{0, s}^{2}\left(\mathbb{R}^{d}\right)=H_{s}^{2}\left(\mathbb{R}^{d}\right)=\{f \mid$ $\left.\widehat{f}(\omega)\langle\omega\rangle^{s} \in L^{2}\left(\mathbb{R}^{d}\right)\right\}$.

(d) Shubin spaces: $M_{s}^{2}\left(\mathbb{R}^{d}\right)=L_{s}^{2}\left(\mathbb{R}^{d}\right) \cap H_{s}^{2}\left(\mathbb{R}^{d}\right)=Q_{s}\left(\mathbb{R}^{d}\right)$ (cf. [37]).

For the results on multiplication and convolution in modulation spaces and in weighted Lebesgue spaces we first introduce the Young functional:

$$
\begin{aligned}
\mathrm{R}(p) \equiv 2-\frac{1}{p_{0}}-\frac{1}{p_{1}}-\frac{1}{p_{2}} & \\
& \\
& p=\left(p_{0}, p_{1}, p_{2}\right) \in[1, \infty]^{3} .
\end{aligned}
$$

When $\mathrm{R}(p)=0$, the Young inequality for convolution reads as

$$
\begin{aligned}
& \left\|f_{1} * f_{2}\right\|_{L^{p_{0}^{\prime}}} \leq\left\|f_{1}\right\|_{L^{p_{1}}}\left\|f_{2}\right\|_{L^{p_{2}}}, \\
& \qquad f_{j} \in L^{p_{j}}\left(\mathbb{R}^{d}\right), \quad j=1,2 .
\end{aligned}
$$

The following theorem is an extension of the Young inequality to the case of weighted Lebesgue spaces and modulation spaces when $0 \leq R(p) \leq 1 / 2$.

Theorem 8. Let $s_{j}, t_{j} \in \mathbb{R}$ and $p_{j}, q_{j} \in[1, \infty], j=0,1,2$. Assume that $0 \leq \mathrm{R}(p) \leq 1 / 2, \mathrm{R}(q) \leq 1$, and

$$
\begin{aligned}
& 0 \leq t_{j}+t_{k}, \quad j, k=0,1,2, j \neq k, \\
& 0 \leq t_{0}+t_{1}+t_{2}-d \cdot \mathrm{R}(p), \\
& 0 \leq s_{0}+s_{1}+s_{2},
\end{aligned}
$$

with strict inequality in (30) when $\mathrm{R}(p)>0$ and $t_{j}=d \cdot \mathrm{R}(p)$ for some $j=0,1,2$.

Then $\left(f_{1}, f_{2}\right) \mapsto f_{1} * f_{2}$ on $C_{0}^{\infty}\left(\mathbb{R}^{d}\right)$ extends uniquely to a continuous map from

(1) $L_{t_{1}}^{p_{1}}\left(\mathbb{R}^{d}\right) \times L_{t_{2}}^{p_{2}}\left(\mathbb{R}^{d}\right)$ to $L_{-t_{0}}^{p_{0}^{\prime}}\left(\mathbb{R}^{d}\right)$;

(2) $M_{s_{1}, t_{1}}^{p_{1}, q_{1}}\left(\mathbb{R}^{d}\right) \times M_{s_{2}, t_{2}}^{p_{2}, q_{2}}\left(\mathbb{R}^{d}\right)$ to $M_{-s_{0},-t_{0}}^{p_{0}^{\prime}, q^{\prime}}\left(\mathbb{R}^{d}\right)$.
For the proof we refer to [24]. It is based on the detailed study of an auxiliary three-linear map over carefully chosen regions in $\mathbb{R}^{d}$; see Sections 3.1 and 3.2 in [24]. This result extends multiplication and convolution properties obtained in [38]. Moreover, the result is sharp in the following sense.

Theorem 9. Let $p_{j}, q_{j} \in[1, \infty]$ and $s_{j}, t_{j} \in \mathbb{R}, j=0,1,2$. Assume that at least one of the following statements holds true:

(1) The map $\left(f_{1}, f_{2}\right) \mapsto f_{1} * f_{2}$ on $C_{0}^{\infty}\left(\mathbb{R}^{d}\right)$ is continuously extendable to a map from $L_{t_{1}}^{p_{1}}\left(\mathbb{R}^{d}\right) \times L_{t_{2}}^{p_{2}}\left(\mathbb{R}^{d}\right)$ to $L_{-t_{0}}^{p_{0}^{\prime}}\left(\mathbb{R}^{d}\right)$.

(2) The map $\left(f_{1}, f_{2}\right) \mapsto f_{1} * f_{2}$ on $C_{0}^{\infty}\left(\mathbb{R}^{d}\right)$ is continuously extendable to a map from $M_{s_{1}, t_{1}}^{p_{1}, q_{1}}\left(\mathbb{R}^{d}\right) \times M_{s_{2}, t_{2}}^{p_{2}, q_{2}}\left(\mathbb{R}^{d}\right)$ to $M_{-s_{0},-t_{0}}^{p_{0}^{\prime}, q_{0}^{\prime}}\left(\mathbb{R}^{d}\right)$;

Then (29) and (30) hold true.

\section{Continuity Properties}

We start estimates of the modulation space norm of the crossWigner distribution (cf. [21]). We refer to [39, Theorem 4.2] for more refined estimates, and note that in [40] the sufficient conditions for the continuity of the cross-Wigner distribution on modulation spaces are proved to be necessary too (in the unweighted case). Proposition 10 coincides with certain sufficient conditions from [40, Theorem 1.1] when restricted to $\mathrm{R}(p)=0, t_{0}=-t_{1}$, and $t_{2}=\left|t_{0}\right|$.

Proposition 10. Let the assumptions of Theorem 8 hold. If $f, g \in \mathcal{S}\left(\mathbb{R}^{d}\right)$, then the map $(f, g) \mapsto W(g, f)$ where $W$ is the cross-Wigner distribution given by (14) extends to sesquilinear continuous map from $M_{t_{2}}^{p_{2}}\left(\mathbb{R}^{d}\right) \times M_{t_{1}}^{p_{1}}\left(\mathbb{R}^{d}\right)$ to $M_{-t_{0}, 0}^{1, p_{0}^{\prime}}\left(\mathbb{R}^{2 d}\right)$.

Proof. We give a short version of the proof for the sake of completeness and refer to [21] for details. Let $f, g \in \mathcal{S}\left(\mathbb{R}^{d}\right)$. Then

$$
\begin{aligned}
& \|W(g, f)\|_{M_{-t_{0}, 0}^{1, p_{0}^{\prime}}} \\
& \quad=\left\|\left(V_{W\left(\psi_{1}, \psi_{2}\right)} W(g, f)\right)(z, \zeta)\langle\zeta\rangle^{-t_{0}}\right\|_{L^{1, p_{0}^{\prime}}},
\end{aligned}
$$

where $z, \zeta \in \mathbb{R}^{2 d}$, since, by Theorem 7(1), modulation spaces are independent of the choice of the window function (from $\left.\mathcal{S}\left(\mathbb{R}^{2 d}\right) \backslash 0\right)$.

From

$$
\begin{array}{r}
W(f, g)(x, \omega)=2^{d} e^{4 \pi i x \cdot \omega} V_{g^{*}} f(2 x, 2 \omega), \\
\quad f, g \in \mathcal{S}^{(1)}\left(\mathbb{R}^{d}\right),
\end{array}
$$


see [26, Lemma 4.3.1], and from the proof of [26, Lemma 14.5 .1 (b)] it follows that

$$
\begin{aligned}
& \left(V_{W\left(\psi_{1}, \psi_{2}\right)} W(g, f)\right)(z, \zeta) \\
& =e^{-2 \pi i z_{2} \zeta_{2}} \overline{V_{\psi_{1}} f}\left(z_{1}+\frac{\zeta_{2}}{2}, z_{2}-\frac{\zeta_{1}}{2}\right) \\
& \cdot V_{\psi_{2}} g\left(z_{1}-\frac{\zeta_{2}}{2}, z_{2}+\frac{\zeta_{1}}{2}\right) .
\end{aligned}
$$

Hence the norms in (32) are equivalent to

$$
\begin{gathered}
\left(\int_{\mathbb{R}^{2 d}}\left(\left|V_{\psi_{1}} f\right| *\left|{\overline{V_{\psi_{2}} g}}^{*}\right|\right)\left(\zeta_{2},-\zeta_{1}\right)\right. \\
\left.\cdot\left\langle\left(\zeta_{2},-\zeta_{1}\right)\right\rangle^{-t_{0} p_{0}^{\prime}} d \zeta\right)^{1 / p_{0}^{\prime}},
\end{gathered}
$$

where the convolution is obtained from the integration over $z$ and after the change of variables $\left(z_{1}, z_{2}\right) \mapsto\left(z_{1}-\zeta_{2} / 2, z_{2}+\right.$ $\left.\zeta_{1} / 2\right)$; see also [13, Proposition 2.5]. Therefore

$$
\begin{aligned}
\|W(g, f)\|_{M_{-t_{0}, 0}^{1, p_{0}^{\prime}}} & \leq\left\|\left|V_{\psi_{1}} f\right| *\left|V_{\psi_{2}} g\right|^{*}\right\|_{L_{-t_{0}}^{p_{0}^{\prime}}} \\
& \leq\left\|V_{\psi_{1}} f\right\|_{L_{t_{1}}^{p_{1}}}\left\|V_{\psi_{2}} g\right\|_{L_{t_{2}}^{p_{2}}},
\end{aligned}
$$

where the last estimate follows from Theorem $8(1)$.

We refer to [10] for the multilinear version of (32), which in turn gives the multilinear version of Corollary 11 (in unweighted case). In fact, from the inspection of the proof of Proposition 10, the definition of $W(\vec{f}, \vec{g})$ given by (17), and (25) we conclude the following.

Corollary 11. Let the assumptions of Theorem 8 hold. If $f_{1}, f_{2}, g_{1}$, and $g_{2} \in \mathcal{S}\left(\mathbb{R}^{d}\right), \vec{f}=\left(f_{1}, f_{2}\right)$, and $\vec{g}=\left(g_{1}, g_{2}\right)$, then the map $(\vec{f}, \vec{g}) \mapsto W(\vec{g}, \vec{f})$, where $W$ is the crossWigner distribution given by (17) which extends to a continuous map from $\mathscr{M}_{t_{2}}^{p_{2}}\left(\mathbb{R}^{d}\right) \times \mathscr{M}_{t_{1}}^{p_{1}}\left(\mathbb{R}^{d}\right)$ to $\mathscr{M}_{-t_{0}, 0}^{1, p_{0}^{\prime}}\left(\mathbb{R}^{2 d}\right)$.

Remark 12. Proposition 10, when restricted to $s=-t_{0}=t_{1}=$ $t_{2} \geq 0, p=p_{0}^{\prime}=p_{2} \in[1, \infty]$, and $p_{1}=1$ gives

$$
\left\|W\left(\varphi_{2}, \varphi_{1}\right)\right\|_{M_{s, 0}^{1, p}} \lesssim\left\|\varphi_{1}\right\|_{M_{s}^{1}}\left\|\varphi_{2}\right\|_{M_{s}^{p}}
$$

that is, [13, Proposition 2.5] (with a slightly different notation).

For a certain choice of the parameters (in particular when $p_{0}^{\prime} \neq p_{2}$ ) we obtain estimates sharper than (37). In particular, if $p_{1}=1, p_{2}=\infty$, and $p \geq 2$, we obtain

$$
\left\|W\left(\varphi_{2}, \varphi_{1}\right)\right\|_{M_{-t_{0}, 0}^{1, p}} \leqslant\left\|\varphi_{1}\right\|_{M_{t_{1}}^{1}}\left\|\varphi_{2}\right\|_{M_{t_{2}}^{\infty}},
$$

with $0<t_{0}+t_{1}+t_{2}-d \cdot \mathrm{R}(p)$ when $p=\infty$ and $t_{j}=d \cdot \mathrm{R}(p)$ for some $j=0,1,2$.

Next we prove an extension of [26, Theorem 14.5.2] to the bilinear Weyl $\Psi D O s$. Recall, if $\sigma \in M^{\infty, 1}\left(\mathbb{R}^{2 d}\right)$ is the
Weyl symbol of $L_{\sigma}$, then [26, Theorem 14.5.2] says that $L_{\sigma}$ is bounded on $M^{p, q}\left(\mathbb{R}^{d}\right), 1 \leq p, q \leq \infty$. This result has a long history starting from the Calderon-Vaillancourt theorem on boundedness of the pseudodifferential operators with smooth and bounded symbols on $L^{2}\left(\mathbb{R}^{d}\right)$, [41]. It is generalized by Sjöstrand in [42] where $M^{\infty, 1}$ is used as appropriate symbol class. Sjöstrand's results were thereafter extended in $[22,26,39,43-45]$. Moreover, we refer to [10-12] for the multilinear Kohn-Nirenberg YDOs.

To deal with duality when $p q=\infty$ we observe that, by a slight modification of [10, Lemma 2.2], the following is true.

Lemma 13. Let $L^{0}\left(\mathbb{R}^{4 d}\right)$ denote the space of bounded, measurable functions on $\mathbb{R}^{4 d}$ which vanish at infinity and put

$$
\begin{aligned}
& \mathscr{M}^{0, q}\left(\mathbb{R}^{2 d}\right)=\left\{f \in \mathscr{M}^{\infty, q}\left(\mathbb{R}^{2 d}\right) \mid V_{\varphi_{1} \otimes \varphi_{2}}\left(f_{1} \otimes f_{2}\right)\right. \\
& \left.\quad \in L^{0}\left(\mathbb{R}^{4 d}\right)\right\}, \quad 1 \leq q<\infty, \\
& \mathscr{M}^{p, 0}\left(\mathbb{R}^{2 d}\right)=\left\{f \in \mathscr{M}^{p, \infty}\left(\mathbb{R}^{2 d}\right) \mid V_{\varphi_{1} \otimes \varphi_{2}}\left(f_{1} \otimes f_{2}\right)\right. \\
& \left.\quad \in L^{0}\left(\mathbb{R}^{4 d}\right)\right\}, \quad 1 \leq p<\infty, \\
& \mathscr{M}^{0,0}\left(\mathbb{R}^{2 d}\right)=\left\{f \in \mathscr{M}^{\infty, \infty}\left(\mathbb{R}^{2 d}\right) \mid V_{\varphi_{1} \otimes \varphi_{2}}\left(f_{1} \otimes f_{2}\right)\right. \\
& \left.\quad \in L^{0}\left(\mathbb{R}^{4 d}\right)\right\},
\end{aligned}
$$

equipped with the norms of $\mathscr{M}^{\infty, q}, \mathscr{M}^{p, \infty}$, and $\mathscr{M}^{\infty, \infty}$, respectively. Then,

(a) $\mathscr{M}^{0, q}$ is $\mathscr{M}^{\infty, q}$-closure of $\mathcal{S}$ in $\mathscr{M}^{\infty, q}$, hence is a closed subspace of $\mathscr{M}^{\infty, q}$. Likewise for $\mathscr{M}^{p, 0}$ and $\mathscr{M}^{0,0}$.

(b) The following duality results hold for $1 \leq p, q<\infty$ : $\left(\mathscr{M}^{0, q}\right)^{\prime}=\mathscr{M}^{1, q^{\prime}},\left(\mathscr{M}^{p, 0}\right)^{\prime}=\mathscr{M}^{p^{\prime}, 1}$, and $\left(\mathscr{M}^{0,0}\right)^{\prime}=$ $\mathscr{M}^{1,1}$.

From now on, we will use these duality relations in the cases $p=\infty$ and/or $q=\infty$ without further explanations.

Theorem 14. Let $\sigma \in M^{\infty, 1}\left(\mathbb{R}^{4 d}\right)$ and let $L_{\sigma}$ be given by (16). The operator $L_{\sigma}$ is bounded from $\mathscr{M}^{p, q}\left(\mathbb{R}^{2 d}\right)$ to $\mathscr{M}^{p, q}\left(\mathbb{R}^{2 d}\right), 1 \leq$ $p, q \leq \infty$, with a uniform estimate $\left\|L_{\sigma}\right\|_{o p} \leq\|\sigma\|_{M^{\infty, 1}}$ for the operator norm.

Proof. Note that the integrals here below are well defined and that the order of integration is irrelevant. We have

$$
\begin{aligned}
& \left|\left\langle L_{\sigma} \vec{f}, \vec{g}\right\rangle\right| \\
& \quad \leq\left\|V_{\Phi} \sigma\right\|_{L^{\infty, 1}\left(\mathbb{R}^{8 d}\right)}\left\|V_{\Phi}(W(\vec{g}, \vec{f}))\right\|_{L^{1, \infty}\left(\mathbb{R}^{8 d}\right)} \\
& \quad=\|\sigma\|_{M^{\infty, 1}\left(\mathbb{R}^{4 d}\right)} \sup _{\zeta \in \mathbb{R}^{4 d}} \int_{\mathbb{R}^{4 d}}\left|V_{\Phi}(W(\vec{g}, \vec{f}))(z, \zeta)\right| d z,
\end{aligned}
$$


where $\Phi \in \mathcal{S}\left(\mathbb{R}^{2 d}\right) \backslash 0$ is a window function. By slight modifications of the proof of [26, Theorem 14.5.2] we obtain the following estimate:

$$
\begin{aligned}
& \int_{\mathbb{R}^{4 d}}\left|V_{\Phi}(W(\vec{g}, \vec{f}))(z, \zeta)\right| d z \\
& \quad \leq\|\vec{f}\|_{\mathscr{M}^{p, q\left(\mathbb{R}^{2 d}\right)}}\|\vec{g}\|_{\mathscr{M}^{p^{\prime}, q^{\prime}\left(\mathbb{R}^{2 d}\right)}},
\end{aligned}
$$

uniformly in $\zeta \in \mathbb{R}^{4 d}$. Therefore,

$$
\begin{aligned}
& \left|\left\langle L_{\sigma} \vec{f}, \vec{g}\right\rangle\right| \\
& \quad \leq\|\sigma\|_{M^{\infty, 1}\left(\mathbb{R}^{4 d}\right)}\|\vec{f}\|_{\mathcal{M}^{p, q}\left(\mathbb{R}^{2 d}\right)}\|\vec{g}\|_{\mathscr{M}^{p^{\prime}, q^{\prime}\left(\mathbb{R}^{2 d}\right)}},
\end{aligned}
$$

for all $\vec{g} \in \mathscr{M}^{p^{\prime}, q^{\prime}}\left(\mathbb{R}^{2 d}\right)$. It follows that $L_{\sigma} \vec{f} \in \mathscr{M}^{p, q}\left(\mathbb{R}^{2 d}\right)$ and

$$
\left\|L_{\sigma} \vec{f}\right\|_{\mathscr{M}^{p, q\left(\mathbb{R}^{2 d}\right)}} \leq\|\sigma\|_{M^{\infty, 1}\left(\mathbb{R}^{4 d}\right)}\|\vec{f}\|_{\mathscr{M}^{p, q}\left(\mathbb{R}^{2 d}\right)} .
$$

Hence the operator $L_{\sigma}$ is bounded on $\mathscr{M}^{p, q}\left(\mathbb{R}^{2 d}\right)$ and $\left\|L_{\sigma}\right\|_{o p} \leq\|\sigma\|_{M^{\infty, 1}}$ as claimed.

Finally, we use the relation between the Weyl pseudodifferential operators and the localization operators (Theorem 4) and the convolution estimates for modulation spaces (Theorem 8 ) to obtain continuity results for $A_{a}^{\varphi}$ for different choices of windows and symbols.

Theorem 15. Let the assumptions of Theorem 8 hold and let $\varphi_{j}, \phi_{j} \in \mathcal{S}^{\prime}\left(\mathbb{R}^{d}\right) j=1,2$, and $\vec{\varphi}=\left(\varphi_{1}, \varphi_{2}\right), \vec{\phi}=\left(\phi_{1}, \phi_{2}\right)$. If $\vec{\varphi} \in \mathscr{M}_{t_{1}}^{p_{1}}\left(\mathbb{R}^{2 d}\right), \vec{\phi} \in \mathscr{M}_{t_{2}}^{p_{2}}\left(\mathbb{R}^{2 d}\right)$, and $a \in M_{u, v}^{\infty, r}\left(\mathbb{R}^{4 d}\right)$, where $1 \leq r \leq p_{0}, u \geq t_{0}$, and $v \geq d \mathrm{R}(p)$ with $v>d \mathrm{R}(p)$ when $\mathrm{R}(p)>0$, then $A_{a}^{\varphi}$ is bounded on $\mathscr{M}^{p, q}\left(\mathbb{R}^{2 d}\right)$ for all $1 \leq p, q \leq$ $\infty$, and the operator norm satisfies the uniform estimate

$$
\left\|A_{a}^{\varphi}\right\|_{o p} \leq\|a\|_{M_{u, v}^{\infty, r},}\|\vec{\varphi}\|_{\mathscr{M}_{t_{1}}^{p_{1}}}\|\vec{\phi}\|_{\mathscr{M}_{t_{2}}^{p_{2}}} .
$$

Proof. Let $\vec{\varphi} \in \mathscr{M}_{t_{1}}^{p_{1}}\left(\mathbb{R}^{2 d}\right)$ and $\vec{\phi} \in \mathscr{M}_{t_{2}}^{p_{2}}\left(\mathbb{R}^{2 d}\right)$. From Corollary 11 it follows that

$$
\begin{aligned}
W(\vec{\phi}, \vec{\varphi}) & =W\left(\phi_{1}, \varphi_{1}\right) \otimes W\left(\phi_{2}, \varphi_{2}\right) \\
& \in \mathscr{M}_{-t_{0}, 0}^{1, p_{0}^{\prime}}\left(\mathbb{R}^{4 d}\right) .
\end{aligned}
$$

Now, the calculation of $a * W(\vec{\phi}, \vec{\varphi})$ from the proof of Theorem 4 together with Theorem 8(2) implies that

$$
a * W(\vec{\phi}, \vec{\varphi}) \in M^{\tilde{p}, 1}\left(\mathbb{R}^{4 d}\right), \quad \widetilde{p} \geq 2,
$$

if the involved parameters fulfill the conditions of the theorem.

On the one hand, for the Lebesgue parameters it is easy to see that $\tilde{p} \geq 2$ is equivalent to $\mathrm{R}(p)=\mathrm{R}(p, \infty, 1) \in[0,1 / 2]$ and that $1 \leq r \leq p_{0}$ is equivalent to $\mathrm{R}(q)=\mathrm{R}\left(\infty, r, p_{0}^{\prime}\right) \leq 1$.
On the other hand, by the choice of the weight parameters $u$ and $v$ it follows that

$$
a *\left(W\left(\phi_{1}, \varphi_{1}\right) \otimes W\left(\phi_{2}, \varphi_{2}\right)\right) \in M^{\tilde{p}, 1}\left(\mathbb{R}^{4 d}\right),
$$

$$
\widetilde{p} \geq 2 \text {. }
$$

In particular, if $\tilde{p}=\infty$ then $a *(W(\vec{\phi}, \vec{\varphi})) \in M^{\infty, 1}\left(\mathbb{R}^{4 d}\right)$. Put $\sigma=a * W(\vec{\phi}, \vec{\varphi})$. Then Theorems 14, 4, and 8 imply that

$$
\begin{aligned}
\left\|A_{a}^{\varphi}\right\|_{o p} & =\left\|L_{\sigma}\right\|_{o p} \leq\|\sigma\|_{M^{\infty, 1}} \\
& \leq\|a\|_{M_{u, v}^{\infty, r}}\|\vec{\varphi}\|_{\mathscr{M}_{t_{1}}^{p_{1}}}\|\vec{\phi}\|_{\mathscr{M}_{t_{2}}^{p_{2}}},
\end{aligned}
$$

and the Theorem is proved.

We remark that a modification of Theorem 15 can be obtained by using [26, Theorem 14.5.6] instead. That result allows symbols $\sigma$ from weighted modulation spaces. We leave for the reader to check how to change the conditions on weight parameters in Theorem 15 in that case.

We finish with a necessary condition. The proof of Theorem 16 is a slight modification of the proof [13, Theorem $4.3]$, and we leave it for the reader. Here below $\|\cdot\|_{S_{\infty}}$ denotes the norm of a bounded operator.

Theorem 16. Let the assumptions of Theorem 8 hold and let $a \in \mathcal{S}^{\prime}\left(\mathbb{R}^{4 d}\right)$. If there exists a constant $C=C(a)>0$ depending only on the symbol $a$ and

$$
\left\|A_{a}^{\varphi}\right\|_{S_{\infty}} \leq C\|\vec{\varphi}\|_{\mathscr{M}_{t_{1}}^{p_{1}}}\|\vec{\phi}\|_{\mathscr{M}_{t_{2}}^{p_{2}}}
$$

for all $\vec{\varphi}=\left(\varphi_{1}, \varphi_{2}\right)$ and $\vec{\phi}=\left(\phi_{1}, \phi_{2}\right)$, with $\varphi_{j}, \phi_{j} \in \mathcal{S}\left(\mathbb{R}^{d}\right) j=$ 1,2 , then $a \in M_{t_{0}, 0}^{\infty, 1}\left(\mathbb{R}^{2 d}\right)$.

\section{The Proof of Theorem 4}

Note first that the integrals here below are absolutely convergent and that changing the order of integration is allowed. Moreover, certain oscillatory integrals are meaningful in $\mathcal{S}^{(1)^{\prime}}\left(\mathbb{R}^{d}\right)$ in a suitable interpretation. For example, if $\delta$ denotes the Dirac distribution, then the Fourier inversion formula in the sense of distributions gives $\int e^{2 \pi i x \omega} d \omega=$ $\delta(x)$, wherefrom $\iint \phi(x) e^{2 \pi i(x-y) \omega} d x d \omega=\phi(y)$, when $\phi \epsilon$ $\mathcal{S}^{(1)}\left(\mathbb{R}^{d}\right)$.

We first rewrite (12) in the form of a kernel operator.

$$
\begin{gathered}
\left\langle A_{a}^{\varphi} \vec{f}, \vec{g}\right\rangle=\iint_{\mathbb{R}^{4 d}} a(x, \omega)\left(\int_{\mathbb{R}^{d}} f_{1}\left(t_{1}\right)\right. \\
\left.\cdot \overline{M_{\omega_{1}} T_{x_{1}} \varphi_{1}}\left(t_{1}\right) d t_{1}\right)\left(\int_{\mathbb{R}^{d}} f_{2}\left(t_{2}\right)\right. \\
\left.\cdot \overline{M_{\omega_{2}} T_{x_{2}} \varphi_{2}}\left(t_{2}\right) d t_{2}\right) \cdot\left(\int_{\mathbb{R}^{d}} \overline{g_{1}}\left(s_{1}\right)\right. \\
\left.\cdot M_{\omega_{1}} T_{x_{1}} \phi_{1}\left(s_{1}\right) d s_{1}\right)\left(\int_{\mathbb{R}^{d}} \overline{g_{2}}\left(s_{2}\right)\right.
\end{gathered}
$$


8

Journal of Function Spaces

$$
\begin{aligned}
& \left.\cdot M_{\omega_{2}} T_{x_{2}} \phi_{2}\left(s_{2}\right) d s_{2}\right) d x d \omega \\
& =\iint_{\mathbb{R}^{2 d}} \int_{\mathbb{R}^{2 d}} f_{1}\left(t_{1}\right) f_{2}\left(t_{2}\right) \overline{g_{1}}\left(s_{1}\right) \overline{g_{2}}\left(s_{2}\right) \\
& \cdot\left(\iint_{\mathbb{R}^{4 d}} a(x, \omega) \cdot \overline{M_{\omega_{1}} T_{x_{1}} \varphi_{1}}\left(t_{1}\right) \overline{M_{\omega_{2}} T_{x_{2}} \varphi_{2}}\left(t_{2}\right)\right. \\
& \left.\cdot M_{\omega_{1}} T_{x_{1}} \phi_{1}\left(s_{1}\right) M_{\omega_{2}} T_{x_{2}} \phi_{2}\left(s_{2}\right) d x d \omega\right) d t d s \\
& =\left\langle k, \overline{f_{1} \otimes f_{2}} \otimes\left(g_{1} \otimes g_{2}\right)\right\rangle,
\end{aligned}
$$

where the kernel $k=k(t, s), t=\left(t_{1}, t_{2}\right), s=\left(s_{1}, s_{2}\right)$, and $t_{1}, t_{2}, s_{1}, s_{2} \in \mathbb{R}^{d}$, is given by

$$
\begin{aligned}
& k(t, s)=k\left(t_{1}, t_{2}, s_{1}, s_{2}\right)=\int_{\mathbb{R}^{4 d}} a(x, \omega) \overline{M_{\omega_{1}} T_{x_{1}} \varphi_{1}}\left(t_{1}\right) \\
& \cdot \overline{M_{\omega_{2}} T_{x_{2}} \varphi_{2}}\left(t_{2}\right) M_{\omega_{1}} T_{x_{1}} \phi_{1}\left(s_{1}\right) \\
& \cdot M_{\omega_{2}} T_{x_{2}} \phi_{2}\left(s_{2}\right) d x d \omega=\int_{\mathbb{R}^{4 d}} a(x, \omega) \\
& \cdot \overline{\left(M_{\omega_{1}} T_{x_{1}} \varphi_{1} \otimes M_{\omega_{2}} T_{x_{2}} \varphi_{2}\right)}(t) \\
& \cdot\left(M_{\omega_{1}} T_{x_{1}} \phi_{1} \otimes M_{\omega_{2}} T_{x_{2}} \phi_{2}\right)(s) d x d \omega .
\end{aligned}
$$

Next, we calculate the convolution $a *\left(W\left(\phi_{1}, \varphi_{1}\right) \otimes\right.$ $\left.W\left(\phi_{2}, \varphi_{2}\right)\right)$. We will use $W\left(f_{2}, f_{1}\right)=\overline{W\left(f_{1}, f_{2}\right)}$ and the following covariance property of the Wigner transform:

$$
\begin{aligned}
& W\left(T_{x_{1}} M_{\omega_{1}} \phi_{1}, T_{x_{1}} M_{\omega_{1}} \varphi_{1}\right)\left(p_{1}, q_{1}\right) \\
& =\int_{\mathbb{R}^{d}} T_{x_{1}} M_{\omega_{1}} \phi_{1}\left(p_{1}+\frac{t}{2}\right) \\
& \overline{T_{x_{1}} M_{\omega_{1}} \varphi_{1}\left(p-\frac{t}{2}\right)} e^{-2 \pi i q_{1} t} d t \\
& =\int_{\mathbb{R}^{d}} e^{2 \pi i\left(p_{1}+t / 2\right) \omega_{1}} \phi_{1}\left(p_{1}+\frac{t}{2}-x_{1}\right)
\end{aligned}
$$

$$
\begin{aligned}
& \cdot e^{-2 \pi i\left(p_{1}-t / 2\right) \omega_{1}} \overline{\varphi_{1}\left(p_{1}-\frac{t}{2}-x_{1}\right)} e^{-2 \pi i q_{1} t} d t \\
& =\int_{\mathbb{R}^{d}} \phi_{1}\left(p_{1}-x_{1}+\frac{t}{2}\right) \\
& \overline{\cdot \varphi_{1}\left(p_{1}-x_{1}-\frac{t}{2}\right)} e^{-2 \pi i\left(q_{1}-\omega_{1}\right) t} d t=W\left(\phi_{1}, \varphi_{1}\right)\left(p_{1}\right. \\
& \left.-x_{1}, q_{1}-\omega_{1}\right) .
\end{aligned}
$$

Let $p=\left(p_{1}, p_{2}\right), q=\left(q_{1}, q_{2}\right)$, and $p_{1}, p_{2}, q_{1}$, and $q_{2} \in \mathbb{R}^{d}$. Then

$$
\begin{aligned}
& a *\left(W\left(\phi_{1}, \varphi_{1}\right) \otimes W\left(\phi_{2}, \varphi_{2}\right)\right)(p, q)=\int_{\mathbb{R}^{4 d}} a(x, \omega) \\
& \text { - } W\left(\phi_{1}, \varphi_{1}\right)\left(p_{1}-x_{1}, q_{1}-\omega_{1}\right) W\left(\phi_{2}, \varphi_{2}\right)\left(p_{2}-x_{2}\right. \text {, } \\
& \left.q_{2}-\omega_{2}\right) d x d \omega=\int_{\mathbb{R}^{2 d}} a(x, \omega) \\
& \cdot W\left(T_{x_{1}} M_{\omega_{1}} \phi_{1}, T_{x_{1}} M_{\omega_{1}} \varphi_{1}\right)\left(p_{1}, q_{1}\right) \\
& \cdot W\left(T_{x_{2}} M_{\omega_{2}} \phi_{2}, T_{x_{2}} M_{\omega_{2}} \varphi_{2}\right)\left(p_{2}, q_{2}\right) d x d \omega \\
& =\int_{\mathbb{R}^{4 d}} a(x, \omega)\left(\iint_{\mathbb{R}^{2 d}} T_{x_{1}} M_{\omega_{1}} \phi_{1}\left(p_{1}+\frac{t_{1}}{2}\right)\right. \\
& \cdot \overline{T_{x_{1}} M_{\omega_{1}} \varphi_{1}}\left(p_{1}-\frac{t_{1}}{2}\right) e^{-2 \pi i q_{1} t_{1}} \cdot T_{x_{2}} M_{\omega_{2}} \phi_{2}\left(p_{2}\right. \\
& \left.+\frac{t_{2}}{2}\right) \overline{T_{x_{2}} M_{\omega_{2}} \varphi_{2}}\left(p_{2}-\frac{t_{2}}{2}\right) \\
& \left.\cdot e^{-2 \pi i q_{2} t_{2}} d t_{1} d t_{2}\right) d x d \omega=\int_{\mathbb{R}^{4 d}} a(x, \\
& \omega)\left(\iint_{\mathbb{R}^{d}} M_{\omega_{1}} T_{x_{1}} \phi_{1}\left(p_{1}+\frac{t_{1}}{2}\right) \overline{M_{\omega_{1}} T_{x_{1}} \varphi_{1}}\left(p_{1}-\frac{t_{1}}{2}\right)\right. \\
& \cdot e^{-2 \pi i q_{1} t_{1}} \cdot M_{\omega_{2}} T_{x_{2}} \phi_{2}\left(p_{2}+\frac{t_{2}}{2}\right) \overline{M_{\omega_{2}} T_{x_{2}} \varphi_{2}}\left(p_{2}\right. \\
& \left.\left.-\frac{t_{2}}{2}\right) e^{-2 \pi i q_{2} t_{2}} d t_{1} d t_{2}\right) d x d \omega,
\end{aligned}
$$

where we have used the commutation relation $T_{x} M_{\omega}=$ $e^{-2 \pi i x \cdot \omega} M_{\omega} T_{x}$.

Now,

$$
\begin{aligned}
& \left\langle L_{a * W(\vec{\phi}, \vec{\varphi})} \vec{f}, \vec{g}\right\rangle=\left\langle L_{a *\left(W\left(\phi_{1}, \varphi_{1}\right) \otimes W\left(\phi_{2}, \varphi_{2}\right)\right)} \vec{f}, \vec{g}\right\rangle=\left\langle a *\left(W\left(\phi_{1}, \varphi_{1}\right) \otimes W\left(\phi_{2}, \varphi_{2}\right)\right), W(\vec{g}, \vec{f})\right\rangle=\int_{\mathbb{R}^{4 d}} a(x, \omega) \\
& \cdot \int_{\mathbb{R}^{4 d}}\left(\int_{\mathbb{R}^{4 d}} M_{\omega_{1}} T_{x_{1}} \phi_{1}\left(p_{1}+\frac{t_{1}}{2}\right) \overline{M_{\omega_{1}} T_{x_{1}} \varphi_{1}}\left(p_{1}-\frac{t_{1}}{2}\right) \cdot M_{\omega_{2}} T_{x_{2}} \phi_{2}\left(p_{2}+\frac{t_{2}}{2}\right) \overline{M_{\omega_{2}} T_{x_{2}} \varphi_{2}}\left(p_{2}-\frac{t_{2}}{2}\right) e^{-2 \pi i \mathscr{F}(t-s) q}\right. \\
& \left.\cdot \overline{g_{1}}\left(p_{1}+\frac{s_{1}}{2}\right) \overline{g_{2}}\left(p_{2}+\frac{s_{2}}{2}\right) f_{1}\left(p_{1}-\frac{s_{1}}{2}\right) f_{2}\left(p_{2}-\frac{s_{2}}{2}\right) d t d s\right) d p d q d x d \omega=\int_{\mathbb{R}^{4 d}} a(x, \omega) \\
& \cdot \int_{\mathbb{R}^{2 d}}\left(\int_{\mathbb{R}^{4 d}} M_{\omega_{1}} T_{x_{1}} \phi_{1}\left(p_{1}+\frac{t_{1}}{2}\right) \overline{M_{\omega_{1}} T_{x_{1}} \varphi_{1}}\left(p_{1}-\frac{t_{1}}{2}\right)\right.
\end{aligned}
$$




$$
\begin{aligned}
& \cdot M_{\omega_{2}} T_{x_{2}} \phi_{2}\left(p_{2}+\frac{t_{2}}{2}\right) \overline{M_{\omega_{2}} T_{x_{2}} \varphi_{2}}\left(p_{2}-\frac{t_{2}}{2}\right) \delta\left(t_{1}-s_{1}\right) \delta\left(t_{2}-s_{2}\right) \\
& \left.\cdot \overline{g_{1}}\left(p_{1}+\frac{s_{1}}{2}\right) \overline{g_{2}}\left(p_{2}+\frac{s_{2}}{2}\right) f_{1}\left(p_{1}-\frac{s_{1}}{2}\right) f_{2}\left(p_{2}-\frac{s_{2}}{2}\right) d t d s\right) d p d x d \omega=\int_{\mathbb{R}^{4 d}} a(x, \omega) \\
& \cdot \int_{\mathbb{R}^{2 d}}\left(\int_{\mathbb{R}^{2 d}} M_{\omega_{1}} T_{x_{1}} \phi_{1}\left(p_{1}+\frac{t_{1}}{2}\right) \overline{M_{\omega_{1}} T_{x_{1}} \varphi_{1}}\left(p_{1}-\frac{t_{1}}{2}\right)\right. \\
& \cdot M_{\omega_{2}} T_{x_{2}} \phi_{2}\left(p_{2}+\frac{t_{2}}{2}\right) \overline{M_{\omega_{2}} T_{x_{2}} \varphi_{2}}\left(p_{2}-\frac{t_{2}}{2}\right) \overline{g_{1}}\left(p_{1}+\frac{t_{1}}{2}\right) \overline{g_{2}}\left(p_{2}+\frac{t_{2}}{2}\right) \\
& \left.\cdot f_{1}\left(p_{1}-\frac{t_{1}}{2}\right) f_{2}\left(p_{2}-\frac{t_{2}}{2}\right) d t\right) d p d x d \omega,
\end{aligned}
$$

where the interpretation of oscillatory integrals is used as mentioned in the beginning of this Section. Finally, the change of variables

$$
\begin{aligned}
& p_{1}+\frac{t_{1}}{2}=v_{1}, \\
& p_{1}-\frac{t_{1}}{2}=u_{1}, \\
& p_{2}+\frac{t_{2}}{2}=v_{2}, \\
& p_{2}-\frac{t_{2}}{2}=u_{2}
\end{aligned}
$$

gives

$$
\begin{aligned}
& \left\langle L_{a *\left(W\left(\phi_{1}, \varphi_{1}\right) \otimes W\left(\phi_{2}, \varphi_{2}\right)\right)} \vec{f}, \vec{g}\right\rangle=\int_{\mathbb{R}^{4 d}} \int_{\mathbb{R}^{4 d}} a(x, \omega) \\
& \cdot \overline{\left(M_{\omega_{1}} T_{x_{1}} \varphi_{1} \otimes M_{\omega_{2}} T_{x_{2}} \varphi_{2}\right)}(u) \\
& \cdot \overline{\left(M_{\omega_{1}} T_{x_{1}} \phi_{1} \otimes M_{\omega_{2}} T_{x_{2}} \phi_{2}\right)(v) \cdot\left(f_{1} \otimes f_{2}\right)(u)} \\
& \cdot \overline{\left(g_{1} \otimes g_{2}\right)}(v) d u d v d x d \omega \\
& =\left\langle k, \overline{f_{1} \otimes f_{2}} \otimes\left(g_{1} \otimes g_{2}\right)\right\rangle,
\end{aligned}
$$

where the kernel $k$ is given by (51). The theorem follows from the uniqueness of the kernel.

\section{Conflicts of Interest}

The author declares that there are no conflicts of interest regarding the publication of this paper.

\section{Acknowledgments}

This research is supported by MPNTR of Serbia, Projects nos. 174024 and DS 028 (TIFMOFUS).

\section{References}

[1] F. A. Berezin, "Wick and anti-wick operator symbols," Mathematics of the USSR-Sbornik, vol. 86, no. 128, pp. 578-610, 1971.

[2] D. Slepian, "Some comments on fourier analysis, uncertainty and modeling," SIAM Review, vol. 25, no. 3, pp. 379-393, 1983.

[3] I. Daubechies, "Time-frequency localization operators: a geometric phase space approach," Institute of Electrical and Electronics Engineers Transactions on Information Theory, vol. 34, no. 4, pp. 605-612, 1988.

[4] J. Ramanathan and P. Topiwala, "Time-frequency localization via the Weyl correspondence," SIAM Journal on Mathematical Analysis, vol. 24, no. 5, pp. 1378-1393, 1993.

[5] L. D. Abreu and M. Dörfler, "An inverse problem for localization operators," Inverse Problems, vol. 28, no. 11, Article ID 115001, p. $16,2012$.

[6] M. Engliš, "Toeplitz operators and localization operators," Transactions of the American Mathematical Society, vol. 361, no. 2, pp. 1039-1052, 2009.

[7] M. Engliš, "An excursion into Berezin-Toeplitz quantization and related topics," vol. 251 of Operator Theory: Advances and Applications, pp. 69-115, Birkhäuser, 2016.

[8] M. W. Wong, Wavelet Transforms And Localization Operators, vol. 136, Birkhäuser, Verlag, Basel, Switzerland, 2002.

[9] P. Boggiatto, A. Oliaro, and M. W. Wong, " $L^{p}$ boundedness and compactness of localization operators," Journal of Mathematical Analysis and Applications, vol. 322, no. 1, pp. 193-206, 2006.

[10] Á. Bényi, K. H. Gröchenig, C. Heil, and K. A. Okoudjou, "Modulation spaces and a class of bounded multilinear pseudodifferential operators," The Journal of Operator Theory, vol. 54, no. 2, pp. 387-399, 2005.

[11] Á. Bényi and K. Okoudjou, "Bilinear pseudodifferential operators on modulation spaces," Journal of Fourier Analysis and Applications, vol. 10, no. 3, pp. 301-313, 2004.

[12] Á. Bényi and K. A. Okoudjou, "Modulation space estimates for multilinear pseudodifferential operators," Studia Mathematica, vol. 172, no. 2, pp. 169-180, 2006.

[13] E. Cordero and K. Gröchenig, "Time-frequency analysis of localization operators," Journal of Functional Analysis, vol. 205, no. 1, pp. 107-131, 2003.

[14] E. Cordero and K. Gröchenig, "Symbolic calculus and Fredholm property for localization operators," Journal of Fourier Analysis and Applications, vol. 12, no. 4, pp. 371-392, 2006. 
[15] E. Cordero and K. A. Okoudjou, "Multilinear localization operators," Journal of Mathematical Analysis and Applications, vol. 325, no. 2, pp. 1103-1116, 2007.

[16] E. Cordero, S. Pilipović, L. Rodino, and N. Teofanov, "Localization operators and exponential weights for modulation spaces," Mediterranean Journal of Mathematics, vol. 2, no. 4, pp. 381-394, 2005.

[17] E. Cordero, S. Pilipović, L. Rodino, and N. Teofanov, "Quasianalytic Gelfand-Shilov spaces with application to localization operators," Rocky Mountain Journal of Mathematics, vol. 40, no. 4, pp. 1123-1147, 2010.

[18] E. Cordero, L. Rodino, and K. Gröchenig, "Localization operators and time-frequency analysis," in Harmonic, Wavelet and PAdic Analysis, pp. 83-110, World Scientific Publishing Company, 2007.

[19] H. G. Feichtinger and K. Nowak, "A first survey of gabor multipliers," in Advances in Gabor Analysis, pp. 99-128, Birkhäuser, 2003.

[20] N. Teofanov, "Gelfand-Shilov spaces and localization operators," Functional Analysis, Approximation and Computation, vol. 7, no. 2, pp. 135-158, 2015.

[21] N. Teofanov, "Continuity and Schatten-von Neumann properties for localization operators on modulation spaces," Mediterranean Journal of Mathematics, vol. 13, no. 2, pp. 745-758, 2016.

[22] J. Toft, "Continuity and Schatten properties for pseudodifferential operators on modulation spaces," in Modern Trends in Pseudo-Differential Operators, vol. 172 of Operator Theory: Advances and Applications, pp. 173-206, Birkhäuser, 2007.

[23] J. Toft, "Continuity and Schatten properties for Toeplitz operators on modulation spaces," in Modern Trends in PseudoDifferential Operators, vol. 172 of Operator Theory: Advances and Applications, pp. 313-328, Birkhäuser, 2007.

[24] J. Toft, K. Johansson, S. Pilipović, and N. Teofanov, "Sharp convolution and multiplication estimates in weighted spaces," Analysis and Applications, vol. 13, no. 5, pp. 457-480, 2015.

[25] H. G. Feichtinger, "Modulation spaces on locally compact abelian groups, Technical Report," in Wavelets and Their Applications, M. Krishna, R. Radha, and S. Thangavelu, Eds., pp. 99140, University Vienna \& Allied Publishers, 2003.

[26] K. Gröchenig, Foundations of Time-Frequency Analysis, Birkhauser, Boston, MA, USA, 2001.

[27] I. M. Gelfand and G. E. Shilov, Generalized Functions II, Academic Press, New York, NY, USA, 1968.

[28] F. Nicola and L. Rodino, Global Pseudo-Differential Calculus on Euclidean Spaces, Pseudo-Differential Operators, vol. 4 of Theory and Applications, Birkhäuser, Verlag, Basel, Switzerland, 2010.

[29] K. Gröchenig and G. Zimmermann, "Hardy's theorem and the short-time Fourier transform of Schwartz functions," Journal of the London Mathematical Society-Second Series, vol. 63, no. 1, pp. 205-214, 2001.

[30] N. Teofanov, "Ultradistributions and time-frequency analysis," in Pseudo-Differential Operators and Related Topics, vol. 164 of Operator Theory: Advances and Applications, pp. 173-192, Birkhöuser, 2006.

[31] J. Toft, "The Bargmann transform on modulation and GelfandShilov spaces, with applications to Toeplitz and pseudodifferential operators," Journal of Pseudo-Differential Operators and Applications, vol. 3, no. 2, pp. 145-227, 2012.

[32] G. B. Folland, Harmonic Analysis in Phase Space, vol. 122 of Annals of Mathematics Studies, Princeton University Press, Princeton, NJ, USA, 1989.
[33] M. W. Wong, Weyl Transforms, Universitext, Springer, New York, NY, USA, 1998.

[34] P. Boggiatto, E. Cordero, and K. Gröchenig, "Generalized antiWick operators with symbols in distributional Sobolev spaces," Integral Equations and Operator Theory, vol. 48, no. 4, pp. 427442, 2004.

[35] J. Toft, A. Khrennikov, B. Nilsson, and S. Nordebo, "Decompositions of Gelfand-Shilov kernels into kernels of similar class," Journal of Mathematical Analysis and Applications, vol. 396, no. 1, pp. 315-322, 2012.

[36] K. Gröchenig, "Weight functions in time-frequency analysis," in Pseudo-Differential Operators: Partial Differential Equations and Time-Frequency Analysis, L. Rodino and M. W. Wong, Eds., vol. 52, pp. 343-366, Fields Institute Communications, 2007.

[37] M. A. Shubin, Pseudodifferential Operators and Spectral Theory, Springer, Verlag, Berlin, Germany, 2nd edition, 2001.

[38] S. Pilipović, N. Teofanov, and J. Toft, "Micro-local analysis in Fourier Lebesgue and modulation spaces: part II," Journal of Pseudo-Differential Operators and Applications, vol. 1, no. 3, pp. 341-376, 2010.

[39] J. Toft, "Continuity properties for modulation spaces, with applications to pseudo-differential calculus, II," Annals of Global Analysis and Geometry, vol. 26, no. 1, pp. 73-106, 2004.

[40] E. Cordero and F. Nicola, "Sharp integral bounds for wigner distributions," International Mathematics Research Notices, pp. 1-29, 2016.

[41] A.-P. Calderón and R. Vaillancourt, "On the boundedness of pseudo-differential operators," Journal of the Mathematical Society of Japan, vol. 23, pp. 374-378, 1971.

[42] J. Sjöstrand, "An algebra of pseudodifferential operators," Mathematical Research Letters, vol. 1, no. 2, pp. 185-192, 1994.

[43] K. Gröchenig and C. Heil, "Modulation spaces and pseudodifferential operators," Integral Equations and Operator Theory, vol. 34, no. 4, pp. 439-457, 1999.

[44] K. H. Gröchenig and C. Heil, "Modulation spaces as symbol classes for pseudodifferential operators," in Wavelets and Their Applications, M. Krishna, R. Radha, and S. Thangavelu, Eds., pp. 151-170, Allied Publishers Private Limited, New Dehli, India, 2003.

[45] J. Toft, "Continuity properties for modulation spaces, with applications to pseudo-differential calculus, I," Journal of Functional Analysis, vol. 207, no. 2, pp. 399-429, 2004. 


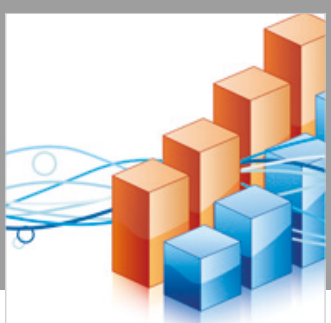

Advances in

Operations Research

\section{-n-m}
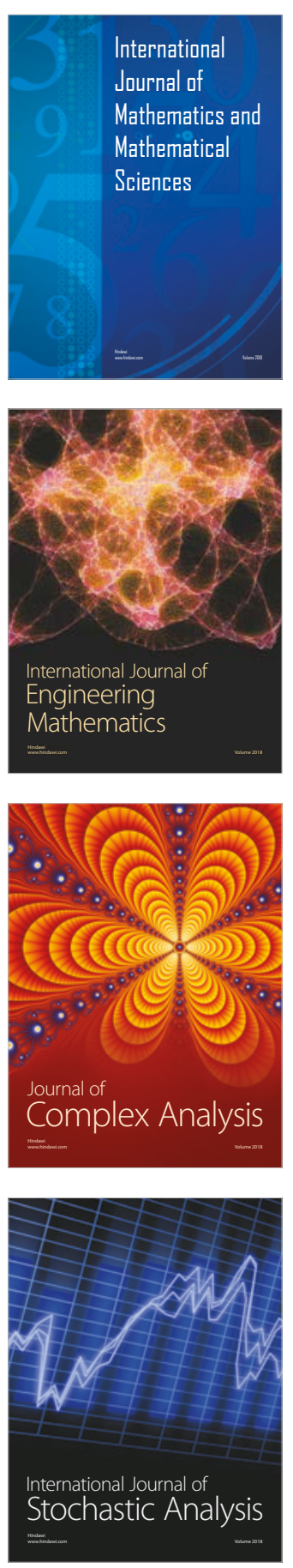
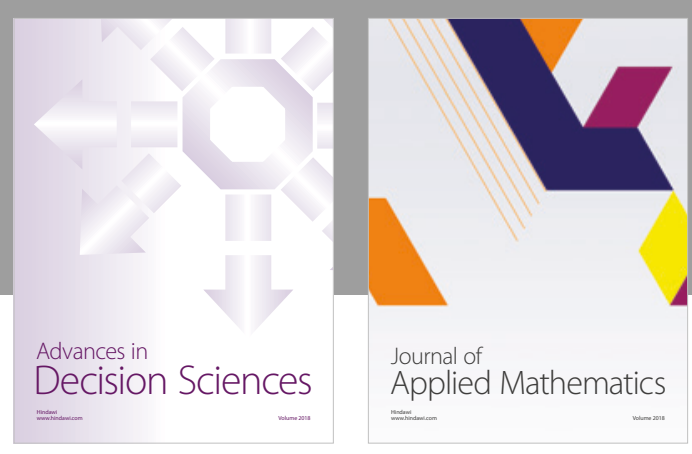

Journal of

Applied Mathematics
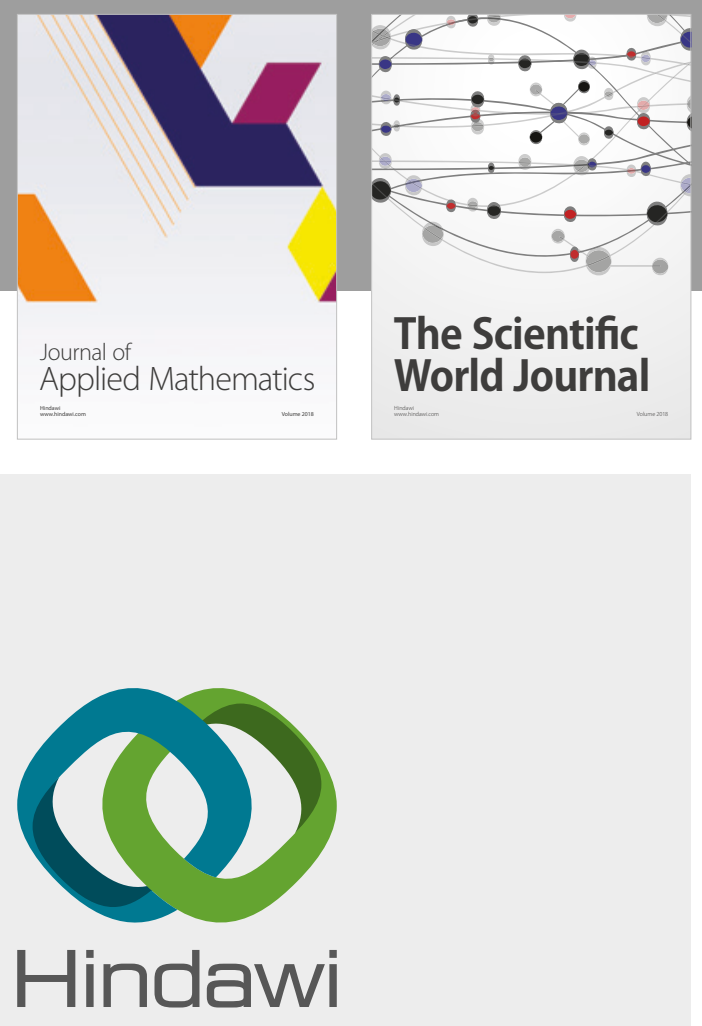

Submit your manuscripts at

www.hindawi.com

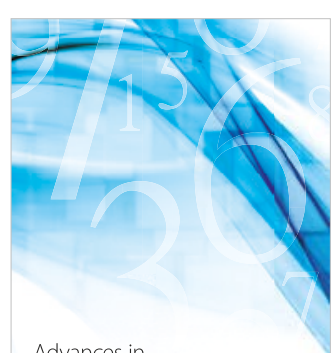

Advances in
Numerical Analysis
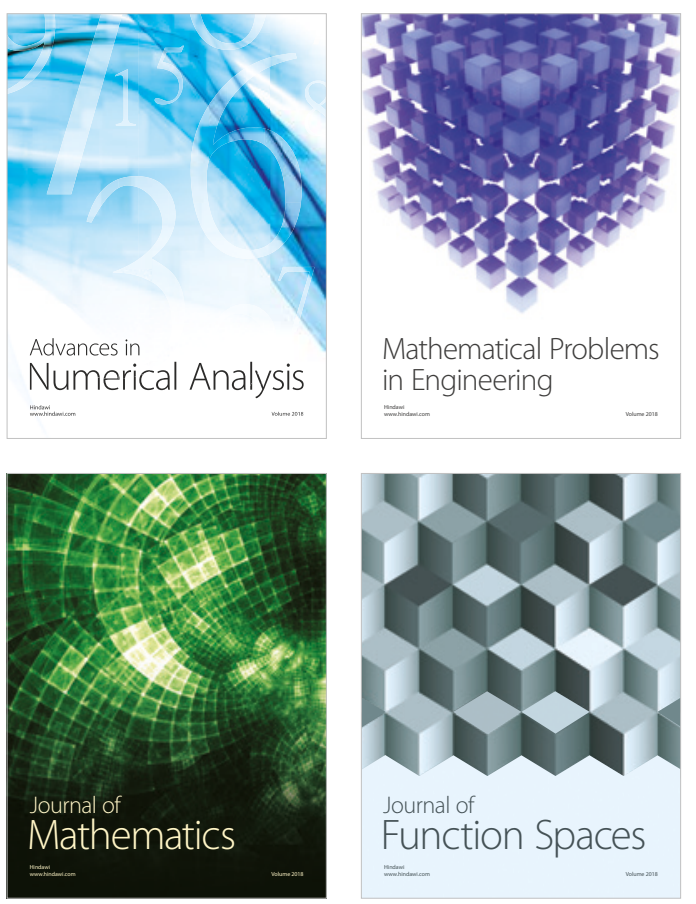

Mathematical Problems in Engineering

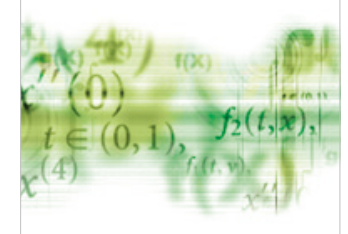

International Journal of

Differential Equations

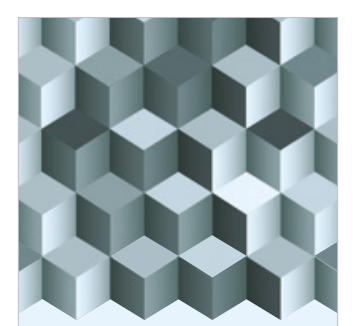

Journal of

Function Spaces

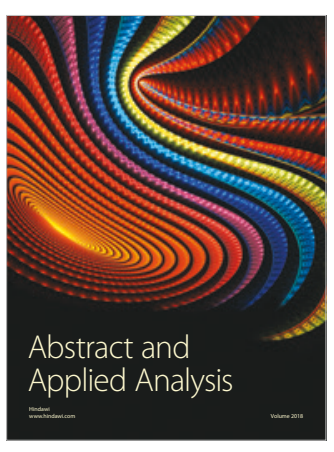

The Scientific

World Journal

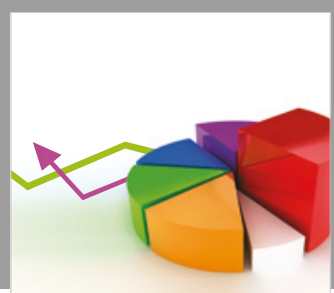

Journal of

Probability and Statistics
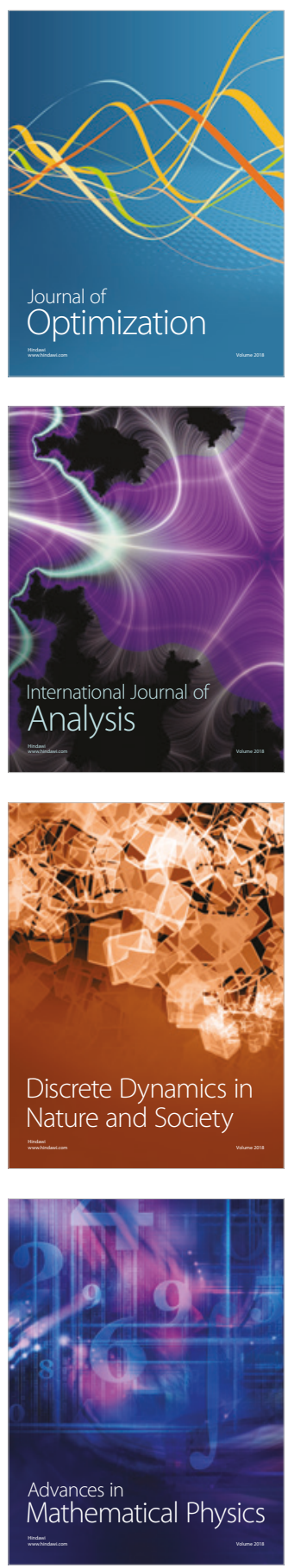